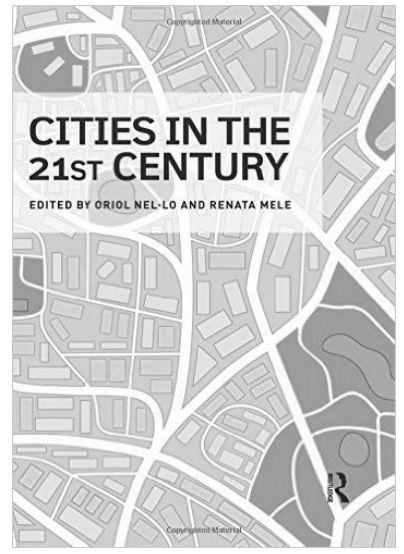

El libro Cities in the 21st Century, editado por Oriol Nel-lo y Renata Mele, es una notable compilación de artículos y casos de estudio, enfocados en las múltiples dimensiones del proceso de urbanización contemporáneo. Con aportes de más de 38 destacados académicos de renombradas universidades y centros de estudio, el libro fue concebido con el propósito de presentar una perspectiva amplia sobre la urbanización mundial, en la que se da cuenta específicamente de la noción de urbanización como un proceso social en el cual convergen múltiples factores. En esa línea, se recopilaron investigaciones de diversas áreas del conocimiento, incluidas geografía, economía, ecología, ingeniería, antropología y ciencias políticas.
CITIES IN THE 2 IST CENTURY [CIUDADES EN EL SIGLO XXI]

Oriol Nel-lo y Renata Mele (eds.)
El libro se divide en seis partes, cada cual apuntando a un aspecto específico de los procesos de urbanización. Dada la diversa naturaleza de los capítulos de este tomo, la estructura propuesta permite al lector organizar la mirada y entender las principales líneas temáticas que el libro busca contener.

La primera parte, "Sistema urbano: Ciudades en la red socioeconómica global", examina la configuración y el funcionamiento de los sistemas urbanos, subrayando la forma en que se han integrado y vuelto más interdependientes a nivel global. La parte 2, "Morfología urbana: Transformaciones y disoluciones", muestra cómo la integración de la economía mundial ha 
estado acompañada de cambios radicales en la morfología urbana, a tal punto que las formas de asentamiento tradicionales, como la ciudad compacta, se comienzan a disolver y formar nuevas configuraciones socioespaciales. $\mathrm{La}$ parte 3, "Ecosistemas urbanos: Recursos y energía", considera los recursos y la energía requeridos para el funcionamiento de la vida urbana, como también la preocupación en torno a su impacto medioambiental. La parte 4, "Tecnologías urbanas: Información, conocimientos, infraestructuras y cultura en las ciudades", destaca la importancia de estos temas para las dinámicas económicas, medioambientales y sociales de las ciudades. La parte 5, "La economía y sociedad urbana: Dinamismo e inequidad", aborda el proceso de urbanización desde una perspectiva económica y social, y analiza la relación entre transformaciones económicas y dinámicas urbanas, dando cuenta de las oportunidades y desafíos asociados a su combinación en la ciudad contemporánea. Finalmente, la parte 6, "Gobernando ciudades: Visiones, proyectos e instrumentos", revisa temas de gobierno urbano, gobernanza urbana y políticas urbanas. Adicionalmente, cada parte está compuesta por tres o cuatro ensayos de algún aspecto del tópico y una serie de casos de estudio, que los editores denominan outlooks. El libro incluye infografías que buscan resumir los temas revisados.

La introducción, de Oriol Nel-lo, entrega un marco teórico-conceptual sintético pero informativo, que invita al lector a mirar los capítulos con un lente que acentúa ciertos fenómenos urbanos de importancia. El apartado describe algunos procesos claves, como el paso de la ciudad amurallada al planeta urbanizado de hoy, el llamado cambio global (incluyendo crecimiento demográfico, calentamiento global y globalización) y sus efectos en la urbanización, la nueva definición de límites de la ciudad, la urbanización como proceso, y las necesidades y dificultades de los gobiernos y políticas urbanas.

Más en detalle, la primera parte se enfoca en analizar las causas y consecuencias de la integración de la red urbana global; dicho fenómeno se ejemplifica en el capítulo escrito por Mireia Belil, que aborda la situación urbana global, aporta datos sobre las conurbaciones de mayor tamaño del mundo y describe los principales desafíos en torno a ellas. Por otra parte, el economista Michael Cohen explica el rol de las ciudades en la globalización de la economía mundial y lo que la interdependencia significa para las finanzas globales, para la manufactura, el intercambio y para las condiciones de vida en las urbes. Julio D. Dávila explora la relación entre el sistema global de ciudades e innovación, como también los desafíos que esos temas plantean en la formación de administradores urbanos. Los casos de estudio de esta parte del libro se enfocan en experiencias de la ciudad de Londres como importante actor en las finanzas globales, el puerto de Shanghái en su rol en el intercambio de bienes manufacturados a escala internacional, y el caso complejo de las migraciones intercontinentales de las ciudades de Ceuta y Melilla en el norte de África.

La segunda parte del libro se enfoca en la nueva morfología de las áreas urbanas, marcada en las últimas décadas por el transporte moderno, el desarrollo de 
infraestructura, cambios en los precios del suelo y segregación de grupos sociales. Esta parte del libro cuenta con aportes de Edward Soja, quien escribe sobre cómo los procesos metropolitanos de las grandes áreas urbanas de hoy engloban regiones completas, arrojando nuevas configuraciones socioespaciales reflejadas tanto en la forma como en la función urbanas. Francesco Indovina se enfoca en la emancipación de las condiciones urbanas en las ciudades europeas. Francesc Muñoz explora nuevas formas de urbanización, incluyendo la urbanalización, que define como paisajes intercambiables con poco significado o valor. Carlos de Mattos, Luis Fuentes y Felipe Link ejemplifican las dinámicas descritas por los tres autores anteriores, usando el caso de la metrópoli de Santiago de Chile. Los casos de estudio de esta parte del tomo plantean ejemplos de nuevas configuraciones urbanas, como la città diffusa del Valle de Po en Italia; los nuevos asentamientos residenciales en Luanda, Angola; el caso de las tensiones entre bazares tradicionales y nuevos centros comerciales en Ankara; y, por último, un caso comparativo entre Buenos Aires y Lima, en que se analizan los cambios de la morfología urbana en los últimos veinte años.

La tercera parte del libro se centra en los temas de ecosistemas urbanos, recursos y energía. Gianluca Bocchi y Angelo Facchini aplican las leyes básicas de los sistemas complejos para entender el metabolismo urbano. Christopher Kennedy muestra cómo las ciudades de hoy se adaptan a los nuevos requerimientos en torno al cambio climático, en especial en relación con la emisión de $\mathrm{CO}^{2}$. Federico Butera se enfoca en los cambios en estilo de vida necesarios para hacer frente al impacto medioambiental de las actividades urbanas, en su contribución al cambio climático. Erik Swyngedouw aborda el tema de la distribución desigual de los costos del cambio climático, que afecta con mayor fuerza a grupos sociales pobres de recursos del sur global. Los casos de estudio muestran ejemplos de infraestructura para el manejo de inundaciones en TokioYokohama, de transporte sustentable en ciudades españolas, y de producción endógena de alimentos en la ciudad de Melbourne, Australia.

La cuarta parte del tomo se enfoca en varias facetas de la relación entre el conocimiento y la tecnología urbana. Franco Farinelli muestra cómo la información y el conocimiento constituyen la base de la economía urbana y que las ciudades en sí son un instrumento que posibilita un acercamiento al conocimiento. Matthew Claudel y Carlo Ratti abordan el tema de la administración urbana mediante tecnologías de la información y su aplicación en los temas de gobernanza y riesgos. Tim Marshall se enfoca en explicar el rol clave de la infraestructura física en el desarrollo de ciudades modernas, ilustrando las dificultades e importancia de su planificación. Richard Plunz y Patricia Culligan muestran la forma en que infraestructuras de diferentes periodos pueden ser reusadas y recicladas, usando el ejemplo de la ciudad de Nueva York. En esta parte del libro también se entrelazan cuatro casos de estudio, incluyendo la concentración de talento intelectual en la ciudad de Boston; la acumulación de datos para la administración urbana en Seattle, Chicago y Lyon; la organización del transporte público en Medellín; y el uso de la tecnología en los servicios públicos en Bari, Italia. 
En la quinta parte del libro se exploran temas relacionados con las dinámicas económicas y sociales en las ciudades. Luis Carvalho y Leo van den Berg discuten el proceso a través del cual las ciudades se han convertido en centros de desarrollo económico, innovación y creatividad. Caroline Moser aborda el tema de la inequidad en las dinámicas de las sociedades urbanas en ciudades del sur global. Joan López escribe sobre la relación entre el desarrollo socioeconómico y la configuración de la red de servicios urbanos en la ciudad contemporánea. Dajian Zhu analiza el caso de las políticas de "nueva urbanización" en China. Los casos de estudio de esta sección incluyen ejemplos tomados del evento masivo World Port Days de Rotterdam; casos de inequidad de género y usos de la ciudad en Ciudad de México; el caso de participación ciudadana en el diseńo y administración de espacios públicos en Barcelona, Glasgow y Nueva York; y, finalmente, el caso de los asentamientos informales en Manila.

Por último, la sexta parte del tomo, con tres capítulos, se enfoca en temas de gobernanza de ciudades y políticas urbanas. El trabajo de Ronan Paddison constituye una reflexión sobre el impacto de nuevas prácticas de gobernanza en las ciudades. Joan Subirats se enfoca en ilustrar las paradojas de la política urbana en el contexto de la globalización económica y la deregularización. João Ferrão se refiere al caso de la Unión Europea y cómo las ciudades pueden desempeñar un rol en la cooperación gubernamental a nivel supranacional. Los casos de estudio de esta sección se refieren a la reciente política de pacificación de las favelas de Río de Janeiro, a los inmigrantes de la ciudad china de
Shenzhen y, por último, al intercambio de desechos por electricidad en la ciudad brasileña de Fortaleza.

En conclusión, esta valiosa compilación constituye un aporte muy significativo a los estudios de los procesos de urbanización contemporáneos. Da cuenta de múltiples temáticas de importancia y relevancia para el futuro desarrollo de zonas urbanas más sustentables, que sean capaces de impartir conocimientos y tecnología para el bien de territorios menos aventajados, con mayor equidad social, más competitivos económicamente y capaces de actuar como catalizadores no solo del desarrollo, sino de la conservación de recursos y suelo en los niveles regionales y nacionales. Adicionalmente, los numerosos casos de estudio que se exponen en este tomo pueden ser considerados potenciales herramientas docentes, al ejemplificar las dinámicas urbanas de la actualidad. De este modo, el libro concentra y permite una revisión fácil, pero a la vez informada y completa, de algunos de los fenómenos urbanos más importantes de la actualidad y activos alrededor del mundo.

\section{Catalina Marshall}

Candidata a Doctor en Arquitectura y Estudios URBANOS

Pontificia Universidad Católica de Chile E-MAIL: CMARSHAL@UC.CL 